\title{
Article
}

http://dx.doi.org/10.11646/phytotaxa.173.4.4

\section{Himalaiella lushaiensis (Asteraceae), a new species from India}

\author{
YOU-SHENG CHEN ${ }^{1 *} \&$ QIAN YUAN ${ }^{1,2}$ \\ 1 State Key Laboratory of Systematic and Evolutionary Botany, Institute of Botany, Chinese Academy of Sciences, 20 Nanxincun, Xiang- \\ shan, Beijing 100093, China \\ 2 University of Chinese Academy of Sciences, Beijing 100049, China \\ *Author for correspondence; e-mail: maple@ibcas.ac.cn
}

\begin{abstract}
Himalaiella lushaiensis (Asteraceae) from India is described and illustrated. It is compared in morphology and distribution with $H$. natmataungensis and H. foliosa. A key to all the species of the genus Himalaiella is provided in aid of their identification.
\end{abstract}

Keywords: Cardueae, Compositae, Mizoram

\section{Introduction}

Himalaiella Raab-Straube (2003: 390), a small, mainly Himalayan genus recently segregated from the species-rich, north temperate Saussurea Candolle (1810: 156), is distinct by the uniseriate pappus, and rugose, muricate or squamulate pericarp with an irregularly toothed crown (Fujikawa \& Ngai 2012). The molecular cladogram resulting from the ITS sequence data indicates that it is more closely related to Jurinea Cassini (1821: 140) than to Saussurea (Raab-Straube 2003). Fourteen species have already been included in Himalaiella, and they are distributed in Afghanistan, Bhutan, SW China, N India, Kashmir, Laos, Myanmar, Nepal, Pakistan, N Thailand, N Vietnam and Iran (Fujikawa et al. 2007; Shi \& Raab-Straube 2011; Fujikawa \& Ngai 2012).

In 2012, in the course of examining specimens at the Herbarium of Royal Botanic Gardens, Kew, U.K. for the preparation of an account of certain genera of Asteraceae for the Flora of Pan-Himalayas, we found a hitherto undescribed species of Himalaiella from India, which is described below. A key to all the species of the genus is provided to facilitate their identification.

Himalaiella lushaiensis Y.S. Chen \& Q. Yuan, sp. nov.

Type:-INDIA. [Mizoram], south of Lushai, open grassland, 1372 m, Febuary 1932, M.L. Werger 422 (holotype K!). Figs. 1, 2 and 3(A $\&$ C).

Annual or biennial herbs. Stem 30-50 cm tall, 3-4 mm in diam., solitary, erect, apically branched, sparsely pubescent. Basal and lower stem leaves petiolate; petiole $2-5 \mathrm{~cm}$ long, base sheathed; leaf blade ovate to elliptic, 11-13 $\times 3.5-$ $4.5 \mathrm{~cm}$, bipinnately divided, abaxially white, conspicuously veined, arachnoid tomentose, gland-dotted, especially so along the veins, adaxially green, scabrous, sparsely strigose, glandular scabrid; primary segments 8-10 pairs, elliptic, apex obtuse and mucronate; secondary lobes 1-3 pairs, obliquely triangular-ovate, sometimes toothed; upper stem leaves sessile, smaller, blade oblong, pinnatisect to pinnately lobed; lateral segments 4-7 pairs, leaf base enlarged and auriculate. Capitula few, usually in a racemiform synflorescence, pedunculate, peduncles $1-6 \mathrm{~cm} \mathrm{long}$. Involucre campanulate, $10-15 \mathrm{~mm}$ in diam. Phyllaries in 5-6 rows, arachnoid, gland-dotted; outer phyllaries oblong, 5-6 $\times$ 1.3-1.5 mm, leafy, gland-dotted, apex densely hairy, obtuse to acute; middle phyllaries oblong, 6-8 8 1.4-1.5 mm, subcoriaceous, deep-brownish hairy, apex acuminate; inner phyllaries narrowly elliptic-linear, deep-brownish hairy, ca. $10 \times 1 \mathrm{~mm}$, apex acuminate (Fig. 4). Receptacle bristles subulate, 4-8 mm long. Corolla purple, 9-10 mm long, gland-dotted, tube 4-5 mm long, limb 6-6.5 mm long, lobes 3-3.5 mm long. Achene brown, ca. $4 \times 1.4 \mathrm{~mm}$, 4-angled, 
Plants with tall stems; capitula numerous, in a racemiform, paniculiform, or corymbose synflorescence...................................... 3

3. Leaf blade shallowly lobed or toothed; outer phyllaries herbaceous H. peguensis [Myanmar, Thailand, China (Guizhou, Yunnan)]

Leaf blade deeply or shallowly lobed; outer phyllaries coriaceous .....

4. Leaf blade sinuately lobed, margin mucronate, abaxially glabrous, sessille; capitula in lax terminal corymbs or solitary axillary ... .. H. chenopodiifolia [Afghanistan, NW Pakistan]

Leaf blade deeply lobed, margin dentate, abaxially white tomentose, petiolate or winged; capitula in sparse racemiform or pa-

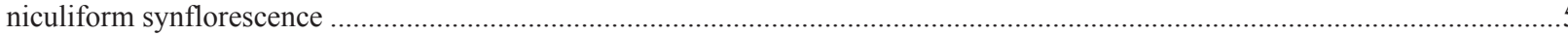

5. Stem profusely branched; phyllaries linear; basal leaves sessile, bipinnate blade with 2-7 pairs of primary segments, segment margin entire or toothed, but never forming secondary lobes.............................................. foilosa [NW India, Nepal, Kashmir] Stem little branched or unbranched; phyllaries oblong, lanceolate or linear; basal leaves petiolate and unwinged; lower leaves petiolate, bipinnate blade with 9-20 pairs of primary segments and with secondary lobes .....

6. Stem little branched; involucre $3-4.5 \mathrm{~cm}$ in diameter; capitula nodding; peduncle $11-30 \mathrm{~cm}$ long; phyllaries linear, apex attenuate or aristate ............................................................................................................... H. natmataungensis [Myanmar] Stem nearly unbranched; involucre $1-1.5 \mathrm{~cm}$ in diameter; capitula erect; peduncle $1-6 \mathrm{~cm}$ long; phyllaries oblong or lanceolate, apex obtuse to acute .................................................................................................................... lushaiensis [NE India]

7. Stem simple, scapiform with reduced leaves; capitulum always solitary; leaf blade adaxially grayish green, arachnoid tomentose. H. abnormis [NW India, W Nepal, China (Tibet)] Stem branched or rarely simple, leafy, not scapiform; capitula numerous or solitary; leaf blade adaxially green, scabrous, strigose, or glabrescent

8. Receptacle with bristles and long subulate paleae; pappus brown; leaf abaxially glabrous

H. auriculata [Bhutan, India, Nepal, Kashmir, China (Tibet)] Receptacle with bristles but without paleae; pappus white or straw-colored; leaf abaxially white hairy or tomentose .................. Plants usually profusely branched, capitula numerous; outer phyllaries with a green, herbaceous appendage..............................10 Plants fewly branched, with 2-4 capitula; outer phyllaries without appendage, long acuminate ..............................................11

10. Involucre 1-2 cm in diameter; capitula solitary or clustered, subsessile or shortly pedunculate; corolla $1-1.2 \mathrm{~cm}$; pappus $0.8-1 \mathrm{~cm}$ ..H. nivea [India, Laos, Myanmar, Nepal, N Thailand, N Vietnam, China (Guizhou, Sichuan, Tibet, Yunnan)] Involucre $2-4 \mathrm{~cm}$ in diameter; capitula mostly solitary, long pedunculate; corolla $1.5-2 \mathrm{~cm}$; pappus $1.4-1.6 \mathrm{~cm}$. ..H. deltoidea [Bhutan, India, Laos, Myanmar, Nepal, Pakistan, Thailand, Vietnam, S China]

11. Leaf blade unlobed, margin dentate H. qinghaiensis [China(Qinghai)]

Leaf blade lyrate lobed, margin entire or toothed

Capitula few, usually 3-9, often long-peduncled, 2-3.5 cm wide; phyllaries greenish; achenes transversely muricate .................... H. heteromalla [Afghanistan, NW Pakistan, N India, Nepal, Bhutan, Kashmir, SE Iran] Capitula usually numerous in corymbs, $0.5-2 \mathrm{~cm}$ wide; phyllaries brownish; achenes smooth or longitudinally ribbed ..............13

13. Capitula cylindric; phyllaries woolly-hairy.............................. H. albescens [Afghanistan, NW Pakistan, N India, Nepal, Kashmir] Capitula campanulate; phyllaries nearly glabrous. H. albescens [Afghanistan, NW Pakistan, N India, Nepal, Kashmir]

14. Leaves mostly basal, usually lyrate-pinnatifid; achene apex faintly dentate .................. H. chitralica [E Afghnistan, NW Pakistan] Leaves sparsely arranged along the stem, usually lanceolate, often pinnately divided, base prominently amplexicaul; achene apex prominently dentate H. afghana [E Afghnistan, NW Pakistan]

\section{Acknowledgements}

We are grateful to curators of the Kew Herbarium for allowing us to study their collections and arranging specimens on loan. We thank Mr. Yun-Xi Zhu for the line illustrations, Dr. K. Fujikawa and an anonymous reviewer for their invaluable comments on the manuscript. This research was supported by the National Natural Science Foundation of China (grant nos. 31370226, 31110103911), "Flora of Pan-Himalayas" Program of Ministry of Science and Technology of the People's Republic of China (grant no. 2013FY112100), and Chinese Academy of Sciences (grant no. GJHZ1140).

\section{References}

Candolle, A.P. de (1810) Observations sur les plantes Composées ou syngenéses (I). Annales du Muséum National d'Histoire Naturelle 16: $135-208$.

Cassini, H. (1821) Proposition d'un nouveau genre de plantes (Jurinea). Bulletin des Sciences, par la Societe Philomatique de Paris 1821: $140-144$.

Edgeworth, M.P. (1846) Descriptions of some unpublished species of plants from north-western India. Transactions of the Linnean Society of London 20: 23-91.

http://dx.doi.org/10.1111/j.1096-3642.1846.tb00410.x 
Fujikawa, K., Kita, Y. \& Ohba, H. (2007) Taxonomic status of Saussurea yakla (Asteraceae) from the Himalayas. Journal of Japanese Botany 82: 130-136.

Fujikawa, K. \& Ngai, S.G. (2012) Taxonomic studies in the Asteraceae of Myanmar II: a new species, Himaleiella natmataungensis (Asteraceae), reported from Natma Taung National Park, Chin State, west-central Myanmar. Makinoa new series 10: $165-171$.

Raab-Straube, E. von (2003) Phylogenetic relationships in Saussurea (Compositae, Cardueae) sensu lato, inferred from morphological, ITS and trnL-trnF sequence data, with a synopsis of Himalaiella gen. nov., Lipschitziella and Frolovia. Willdenowia 33: $379-402$.

Shi, Z. \& Raab-Straube, E. von (2011) Saussurea group. In: Wu, Z.Y. \& Raven, P.H. (Eds.) Flora of China 20-21. Science Press, Beijing and Missouri Botanical Garden Press, St Louis, pp. 42-149.

Susanna, A. \& Garcia-Jacas, N. (2007) Tribe Cardueae Cass. In: Kadereit, J.W. \& Jeffrey, C. (Eds.) The Families and Genera of Vascular Plants 8. Springer, Berlin, Heidelberg \& New York, pp. 123-146. 